\title{
4 A Theoretical Example: CGE Model and Double-Dividend (DD)-Oriented Policies
}

In this section we will present a theoretical non-extensive cross-entropy (NCE) model to estimate parameters of an environmentally extended CGE model to assess impact of the DD hypothesis. The main issue to be underscored remains the rationale for applying the NCE approach in place of the traditional Kullback-Leibler cross-entropy model (Go et al., 2015). The response lies in the statistical properties of power lawrelated NCE. Due to the estimated parameters of constant elasticity of production models, outputs from both techniques must diverge with a higher performance in the case of NCE estimator (see V.5.3 for details). Both solutions will be similar only when the modelled phenomena display Gaussian distribution.

As presented earlier in this part of the book, the negative externalities resulting from pollution is one of the economy-distorting factors that prevents reaching a general equilibrium and Pareto optimum. Pearce (1991) proposed a model to generate double positive impact by introducing a tax imposition on pollutant activities. Once again, the first positive impact results in reducing pollutants through the increase of their production cost. If we use income generated by the tax imposition on pollutant goods to reduce tax in other sectors, a DD may result. This section presents a theoretical CGE model in the context of DD hypothesis testing. The proposed model can enable assessing to what extent the carbon tax can be identified as an important factor affecting the size of the DD, identifying the existence of a strong DD in the economy, or highlighting the weight of certain factors in affecting the presence and size of the DD.

This CE formulation may be written as follows:

$$
\begin{aligned}
& \text { Min } \underset{p, r}{\longrightarrow} \alpha_{1} \frac{1}{q-1} \sum_{k} \sum_{m} p^{E}{ }_{k m}\left[\left(p_{k m}^{E}\right)^{q-1}-\left(q^{E}{ }_{k m}\right)^{q-1}\right]- \\
& \sum_{k} \sum_{m}\left(p^{E}{ }_{k m}-q_{k m}^{E}\right)\left(q^{E}{ }_{k m}\right)^{q-1}+ \\
& \alpha_{2} \frac{1}{q-1} \sum_{t} \sum_{n} \sum_{j} r_{t n j}{ }^{E}\left[\left(r_{t n j}{ }^{E}\right)^{q-1}-\left(s_{t n j}^{E}\right)^{q-1}\right]-\sum_{t} \sum_{n} \sum_{j}\left(r_{t n j}{ }^{E}-s^{E}{ }_{t n j}\right)\left(s^{E}{ }_{t n j}\right)^{q-1}
\end{aligned}
$$

Subject to:

$F\left(X_{t}^{E}, Z_{t}^{0 E}, Z_{t}^{u E}, B^{E}, \delta^{E}\right)=0, \quad \forall t \in T$

$\delta^{E}=P\left(Z_{t}^{E}, B^{E}\right)$

$Y_{t}^{E}=G\left(X_{t}^{E}, Z_{t}^{O E}, Z_{t}^{u E}, B^{E}, \delta^{E}\right)+e_{t} \quad \forall t \in T$ 


$$
\begin{aligned}
& B_{k}^{E}=\sum_{m=1}^{M} p_{k m}^{E} v_{k m} \quad \forall k \in K \\
& e_{t n}^{E}=\sum_{j=1}^{J} r_{t n j}{ }^{E} w_{t n j} \quad \forall t \in T, n \in N \\
& \sum_{m=1}^{M} p_{k m}^{E}=1, \quad \forall k \in K \\
& \sum_{j=1}^{J} r_{t n j}{ }^{E}=1, \quad \forall t \in T, n \in N .
\end{aligned}
$$

The above formulation (5.11a) is the same as the one presented in Part V (Equation 5.11), the only difference being the introduction in the model of an environmental sector. Consequently, this suggests the extension of the pre-existing model by adding environmental relations, as shown in the above optimization system where variables and parameters bear the superscript " $E$ ".

Depending on the target of the environmental model, the above CGE block F(..) $=0$ will include additional endogenous variables (e.g., emitted $\mathrm{CO}_{2}$, the dirty commodity, and its prices), exogenous variables like the environmental tax, the behavioral parameters like the elasticity of the environmental input, etc.

For example, to show the impact of the DD policy, one can add in the above CGE system $F(.)=$.0 the next equations, see $\left(\right.$ Sasmaz, 2016) ${ }^{60}$ for counterfactual simulation purposes:

$$
\mathrm{CO}_{2 t}=\alpha_{1 t}+\beta_{1} \text { envtax }_{t}+\beta_{2} G D P_{t}+\beta_{3} \text { enecons }_{t}+\zeta_{1 t}
$$

$\mathrm{U}$ nemp $_{t}=\alpha_{2 t}+\beta_{4}$ envtax $_{t}+\beta_{5} G D P_{t}+\zeta_{2 t}$

Where $\mathrm{CO}_{2 t}$ (Equation 6.1) is an endogenous variable which depends on the environmental tax rate envtax $x_{t}$ the overall level of gross domestic production $G D P_{t}$, and the

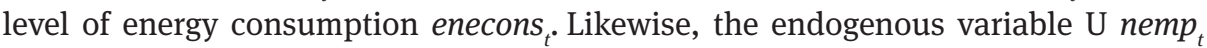
(Equation 6.2) explains the rate of unemployment which depends on the envtax $x_{t}$ and the $G D P_{t}$. The index of time $t$ is related to the targeted time series elements of the environmentally extended social accounting matrices $Y_{t}^{E}$. Parameters $\alpha_{1 t}$ and $\alpha_{2 t}$ are the constants. Parameters $\beta_{j}$ (with $j=5$ ) explain the long-run marginal change of the respective endogenous variable induced by a unit change of the explicative variable. Indeed, parameter interpretation in the model explains a long-run marginal change since we are dealing with an entropy model, the estimates of which will be generated by the maximum entropy principal rule under the CGE constraints. As noted in the previous section where the question was posed as to whether or not the maximum entropy solution is Pareto optimum, the obtained model solution may be different

60 Note that author has checked the DD hypothesis through an econometric panel data model. 
from the solution of the traditional CGE optimal computation, which is not based upon a probabilistic distribution.

Besides the proposed illustrative model in (6.1-6.2), there exists a large formulation of the DD policy-oriented through CGE models (e.g., (Frasera \& Waschik, 2013) (Takeda, 2007), (Taheripour, Khanna i Nelson, 2008), (Bento \& Jacobsen, 2007)). As an interesting case study, Fraser and co-author presented in (Frasera \& Waschik, 2013) a CGE model to assess a DD hypothesis where three taxes were examined: tax on the production of energy goods, on the production of carbon, and on the usage of carbon. To show the existence of DD for each tax revenue raised, authors proposed to offset pre-existing distortions in the CGE model through an endogenous tax that adjusts to keep constant government revenue. Finally, the author's outputs led to the existence of a strong DD associated with the existence of specific (immobile) factors in the production of energy goods. Note that these outputs were generated through a traditional CGE model. 